\title{
Periprothetische Frakturen
}

\author{
Herbert Hessler, Hans-Jörg Oestern
}

\section{Zusammenfassung}

Mit steigender Anzahl von implantierten Endoprothesen nimmt auch die Anzahl periprothetischer Frakturen zu. Die Inzidenz beträgt derzeit ungefähr $0,5-1 \%$ bei Erstimplantaten und etwa das 7-8fache bei Revisionsimplantaten, sie ist bei zementfreier Schaftversorgung höher als bei zementierter Version. Die Klassifikationen nach Bethea bzw. die Vancouver-Klassifikation sind eine gute Basis für die Auswahl differenzierter Therapieverfahren. Bei der zementfreien Implantation können periprothetische Frakturen bereits im ersten Jahr nach der Operation auftreten, bei der zemen- tierten Version erst nach 5 Jahren. Therapeutisch sollte bei fest verankerter Prothese eine Plattenosteosynthese (konventionell oder LISS System) durchgeführt werden, bei Prothesenlockerung ist die Revisionsprothese die Methode der Wahl. In den eigenen Ergebnissen mit 124 periprothetischen Frakturen wurde die Revisionsprothese 55-mal angewandt, 64-mal wurde die Fraktur durch Osteosynthesen stabilisiert. Präventiv sollte eine sorgfältige Schaftpräparation erfolgen, eine intraoperative Röntgenkontrolle durchgeführt werden sowie bei Wechseloperation mit transfemoralem $\mathrm{Zu}-$ gang eine distale prophylaktische Drahtcerclage angelegt werden.

\section{Einleitung}

Die periprothetische Fraktur gewinnt zunehmend an Bedeutung. Hierfür ist vor allem die demografische Entwicklung der Bevölkerung mit Zunahme der alten und sehr alten Menschen verantwortlich. Mit jährlich steigender Anzahl von Erstimplantationen an Hüft- und Knieendoprothesen steigt auch sukzessive die Zahl der periprothetischen Frakturen.

Erfreulicherweise stehen dem Chirurgen heute eine größere Palette operativer Möglichkeiten der Frakturversorgung zur Verfügung.

\section{Inzidenz}

Die Erstbeschreibung der periprothetischen Fraktur erfolgte 1954 durch Horwitz und Lenobel. Seitdem wurden verschiedene Studien publiziert (Tab.1).

OP-JOURNAL 2005; 21: 120-127

(C) Georg Thieme Verlag KG Stuttgart · New York
Die Inzidenz der periprothetischen Fraktur wird unterschiedlich beschrieben. Scott [22] schätzte sie 1975 auf $0,1 \%$. Lowrey [15] gab sie 1991 in einer Serie von 198 implantierten Hüftendoprothesen bei Gebrauch von rigiden Raspeln mit 2,5\% an. Schwartz [21] kam bei der Untersuchung von 1318 zementfreien Hüftendoprothesen auf eine Quote von $3 \%$. Beim Auftreten einer intraoperativen periprothetischen Fraktur fand er eine Schwankungsbreite von $4,1-27,8 \%$ bei zementfreien Hüften, bei zementierten Hüftendoprothesen lag die Rate unter $3 \%$. Beals und Tower [1] schätzten diese auf unter $1 \%$. Nur etwa die Hälfte aller periprothetischen Frakturen wird bei der Entstehung bekannt, die meisten sind unvollständig. In der Mayo-Klinik wurde die intraoperative Frakturhäufigkeit bei Primärinplantationen mit $1 \%$ angegeben (238 Frakturen bei 23980 Patienten). Bei Revisionseingriffen stieg die intraoperative Frakturquote auf 7,8\% (497 bei 6349 Patienten). In der gleichen Klinik wurden postoperative Frakturen in 0,12,1\% der Fälle berichtet, wobei die Quote bei Erstimplantation 1\% und bei Revisionsoperationen $4 \%$ betrug [2].

\section{Klassifikation}

Verschiedene Klassifikationen der periprothetischen Frakturen wurden beschrieben, die sich im Wesentlichen nach der Lokalisation richten [1,17]. Als klinisch praktikabel erweisen sich die Klassifikationen nach Bethea [2] und Duncan [5,28] (Vancouver-Klassifikation) (Abb.1 und 2). Eine erweiterte Version wurde von Fink [8] veröffentlicht.

\section{Ätiologie}

Die häufigste Ursache einer periprothetischen Fraktur ist das Trauma.
Tab. 1 Anzahl und Zeitraum der von den Autoren versorgten periprothetischen Frakturen

\begin{tabular}{llccr} 
& & & Patienten & Zeitraum \\
\hline Gliatis & $(2005)$ & {$[10]$} & 30 & 3 Jahre \\
Bezwada & $(2004)$ & {$[4]$} & 30 & 3 Jahre \\
Parvizi & $(2004)$ & {$[20]$} & 123 & 10 Jahre \\
Spitaler & $(2003)$ & {$[24]$} & 43 & 4 Jahre \\
Haddad & $(2002)$ & {$[11]$} & 40 & 2,5 Jahre \\
Wahl & $(2001)$ & {$[27]$} & 15 & 7 Jahre \\
MacDonald & $(2001)$ & {$[16]$} & 14 & 8 Jahre \\
Kamimeni & $(1999)$ & {$[14]$} & 15 & 7 Jahre \\
Siegmeth & $(1998)$ & {$[23]$} & 51 & 11 Jahre \\
Eingartner & $(1997)$ & {$[7]$} & 12 & 5 Jahre \\
Hopf & $(1996)$ & {$[12]$} & 50 & 10 Jahre
\end{tabular}



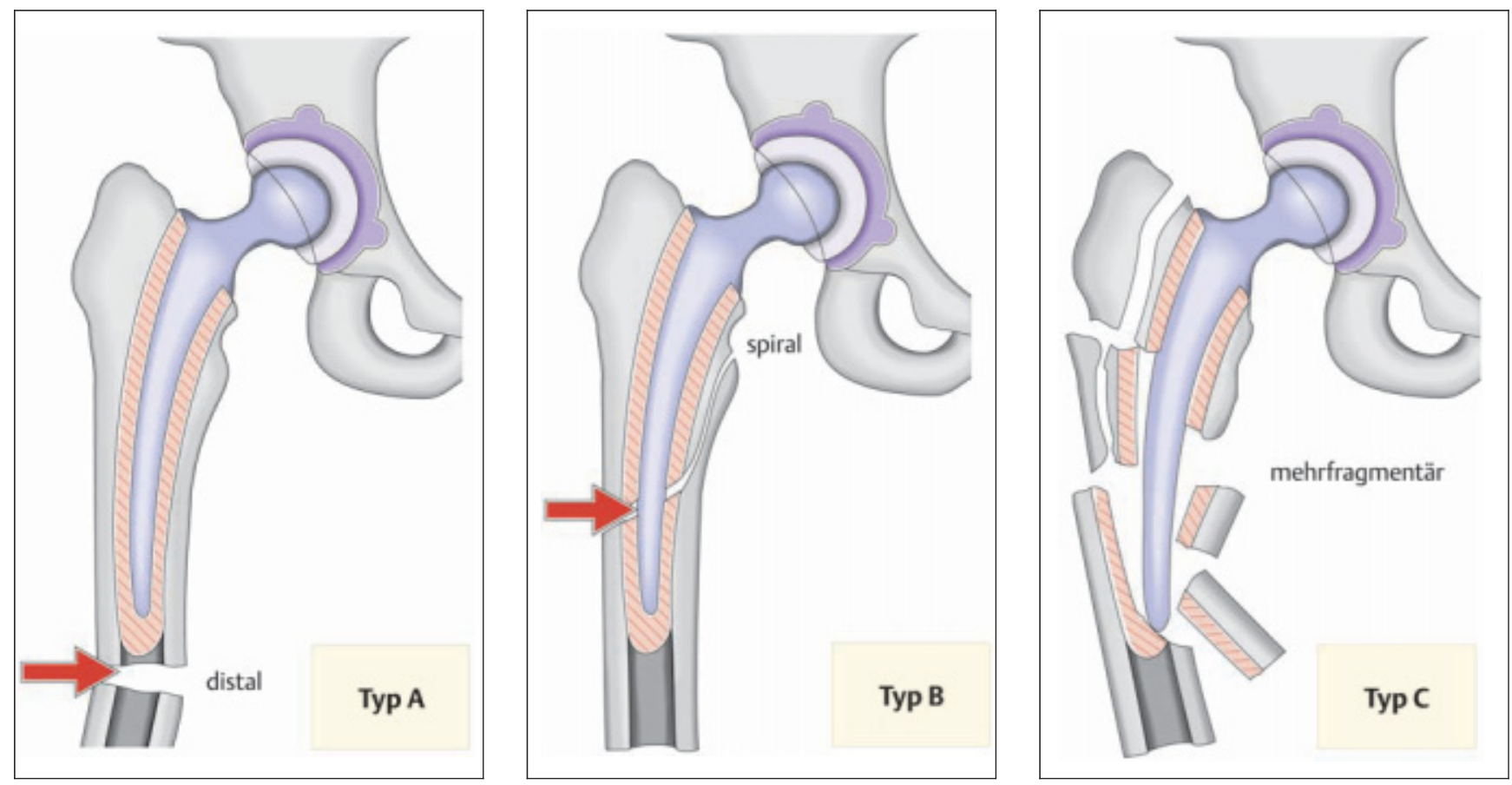

Abb.1 Klassifikation der periprothetischen Fraktur nach Bethea.

Beals und Tower [1] berichten im eigenen Krankengut von 66\% häuslichen Stürzen und $18 \%$ Stürzen außerhalb. In $8 \%$ lag eine Spontanfraktur vor, weitere $8 \%$ hatten ein größeres Trauma erlitten. Weitere Faktoren der periprothetischen Fraktur sind Implantatlockerung, häufig einhergehend mit Polyäthylen induzierten Kortikalisdefekten bzw. mehr oder minder ausgedehnten Osteolysen entlang des Prothesenschaftes oder auch distal davon. Diese Osteolysen schwächen die Stabilität des Implantates, führen konsekutiv zur weiteren Lockerung und stellen potenzielle Sollbruchstellen dar. Eine begleitende Osteoporose erhöht die Frakturgefährdung.

\section{Wann kommt es zur periprothetischen Fraktur?}

Beals und Tower fanden, dass es bei der nicht gelockerten zementierten Hüftendoprothese nach etwa 5,3 Jahren und bei der gelockerten zementierten Hüftendoprothese nach etwa 8,5 Jahren zur periprothetischen Fraktur kommt. Bedingt durch kortikale stress riser [15] bei der Erstimplantation finden sich periprothetische Frakturen bei zementfreien Implantaten bereits nach etwa 4-5 Monaten.

\section{Operative Verfahren}

Die unterschiedlichen Frakturformen benötigen ein differenziertes, individuell ausgewogenes Therapieverfahren: Festverankerte Schaftprothesen können mittels Plattenosteosynthese (Abb.11) ggf. in Kombination mit kortikalen Allografts [29] stabilisiert werden. Im Einzelfalle [6] kommen Drahtcerclagen bzw. mediale Spongiosaplastiken zur Anwendung (Abb.3). Eine gelockerte Schaftendoprothese sollte durch intramedulläre Schienung, wie z.B. mit einer langschaftigen Revisionsprothese mit der Möglichkeit der distalen Verriegelung stabilisiert werden (Abb. 4 u. 12). Im angloamerikranischen Raum ist darüber hinaus bei Prothesenwechseln „impaction grafting“ sehr populär [25]. Distale Femurfrakturen können mittels retrogradem Marknagel [10], teilweise auch in Kombination mit submuskulär eingeschobener Platte (Abb.5) bzw. LISS-System [19] suffizient stabilisiert werden (Abb. 7 u. 10). Die Gefahren der Plattenosteosynthese liegen in vermehrter Deperiostierung, fehlender medialer Abstützung und erschwerter Schraubenverankerung. Bei großvolumigen Femurimplantaten ist bei Verwendung von submuskulär eingeschobenen LISS-Platten monokortikale Stabilisation in Höhe des Endoprothesenschaftes möglich (Abb.8). Allgemeine Probleme bei operativer Versorgung von periprothetischen Frakturen sind Osteoporose, Compliance des Patienten, Alter sowie Begleiterkrankungen und individuelle Operationsfähigkeit.

\section{Ergebnisse (Literatur)}

Eine Metaanalyse von 1980-2005 [18] ergab bei 1615 Patienten 412 Komplikationen, dies entspricht einer Komplikationsrate von $25 \%$. Beals [1] hat bei $93 \mathrm{~Pa}$ tienten von $32 \%$ sehr guten, $16 \%$ guten und 52\% schlechten Ergebnissen berichtet (1996). Die wenigsten Komplikationen bei der Versorgung einer periprothetischen Fraktur fanden sich bei zementfreier Revisionsprothese (20\%). Mehr als doppelt so hoch, nämlich $43 \%$ war die Komplikationsrate bei der Plattenosteosynthese [1]. Die zementierte Revisionsprothese lag mit $61 \%$ noch über der konservativen Frakturbehandlung, die eine Quote von 56\% aufwies. Es fanden sich an Komplikationen bei Plattenosteosynthese in $7 \%$ mäßiggradige Deformierungen, in $10 \%$ eine Pseudarthrose, in 13\% eine erneute Fraktur und in 10\% Fixationsversagen. Parvizi [20] gibt $18 \%$ Komplikationen an. Unter 31 Fällen wurden von Schwartz [21] folgende Komplikationen veröffentlicht:

1 Infekt, 1 Luxation, 6 Plattenlockerungsbrüche und $4 \times$ Exitus letalis. Er schlägt den Andocknagel vor. Wagner [26] konnte mit der Femurrevisionsprothese die Komplikationsrate deutlich senken. Foster [9] berichtet, bei 174 zementierten Hüftendoprothesen keine Fraktur beobachtet zu haben, hingegen 5 Frakturen $(7 \%)$ bei 70 zementfrei 


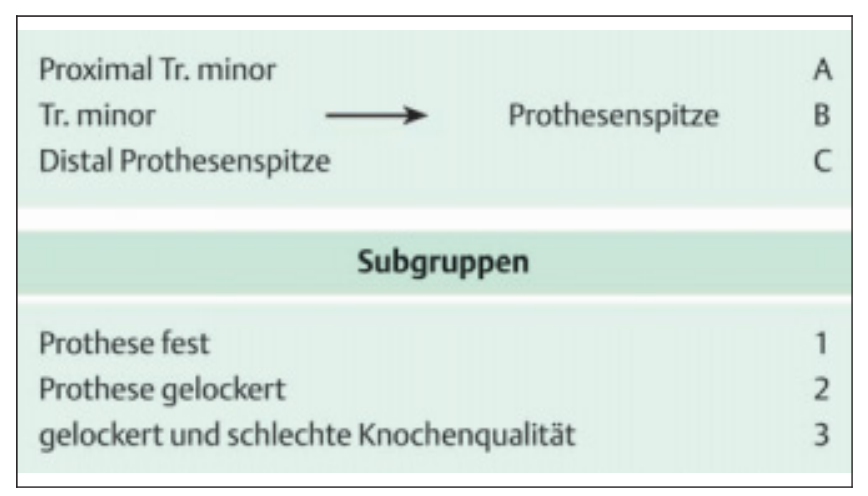

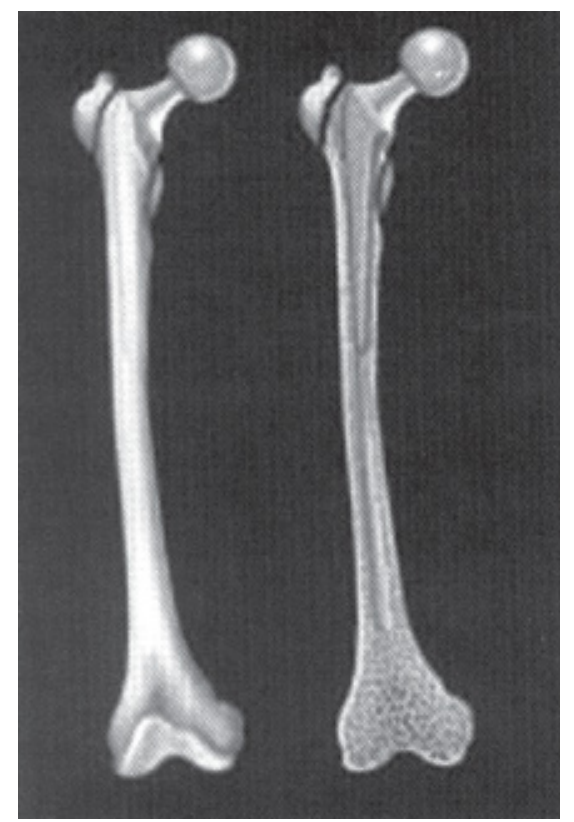

A

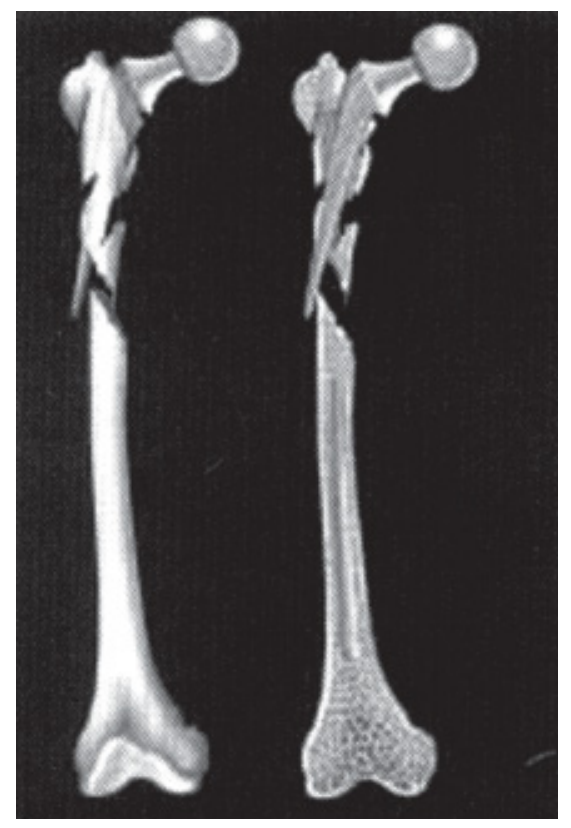

B3

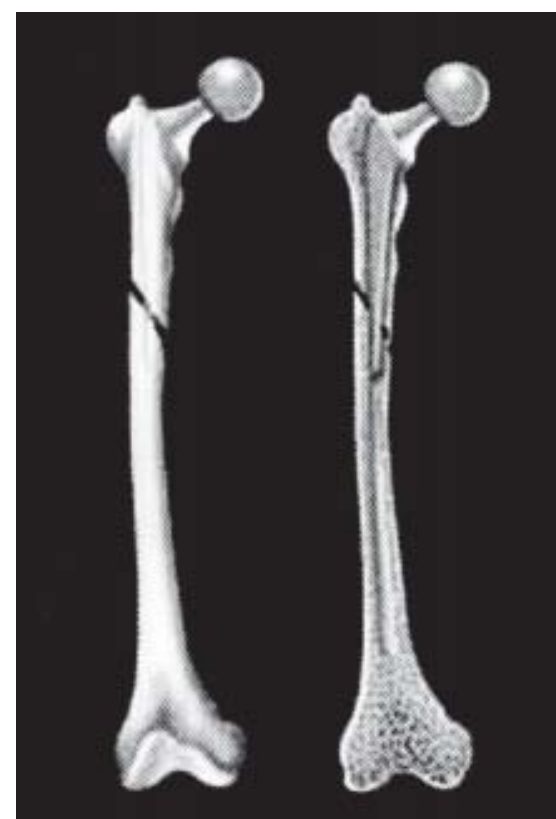

B1

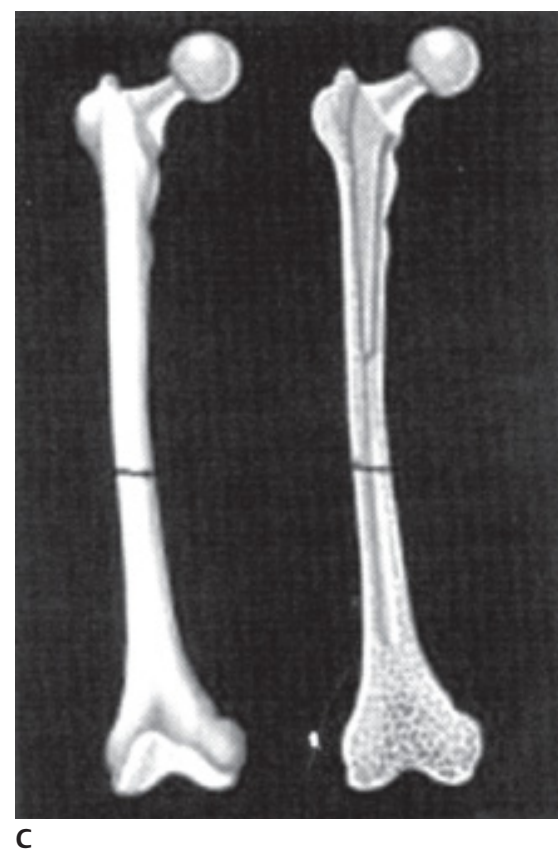

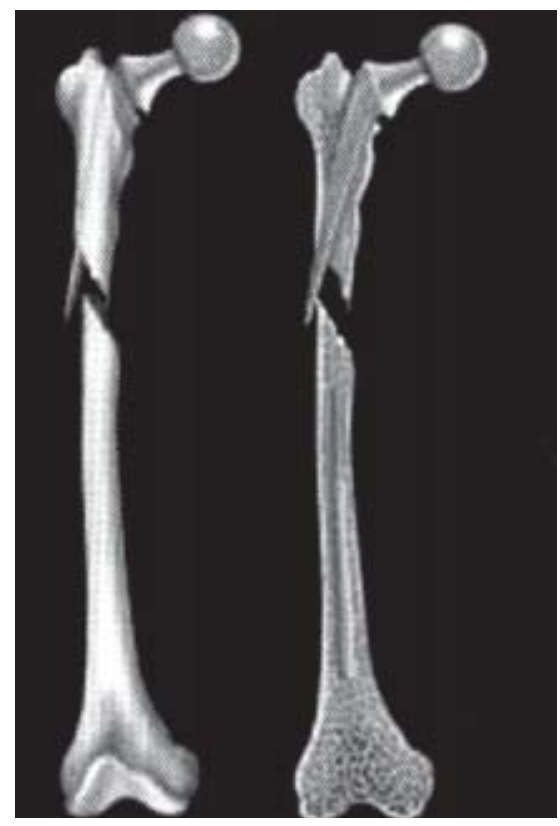

B2

Abb.2 Klassifikation der periprothetischen Fraktur nach Duncan (Vancouver-Klassifikation). 
a, b
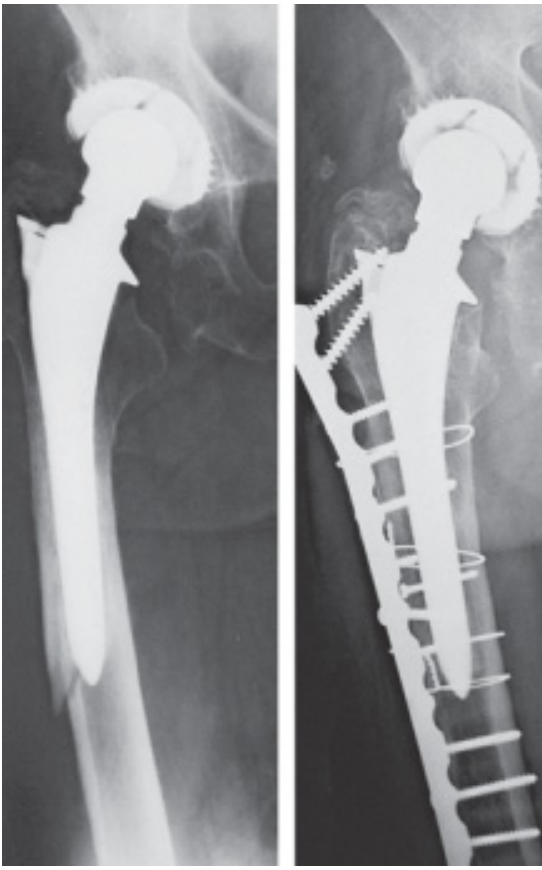

Abb.3 (a) Nach Treppensturz bei 77-jähriger Patientin mit festsitzender Hüftprothese (b) Stabilisation mit Platte u. Cerclagen.

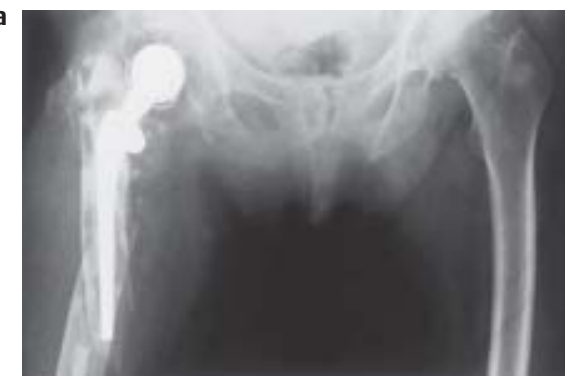

b

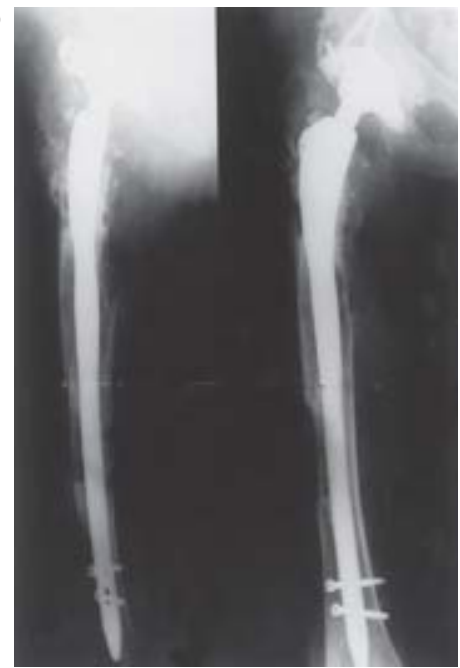

Abb.4 (a) 89-jährige Patientin nach Sturz bei Schaft- und Pfannenlockerung und hochgradiger Knochenatrophie (b) Frakturstabilisation mit distal verriegelter Revisionslangschaftprothese.
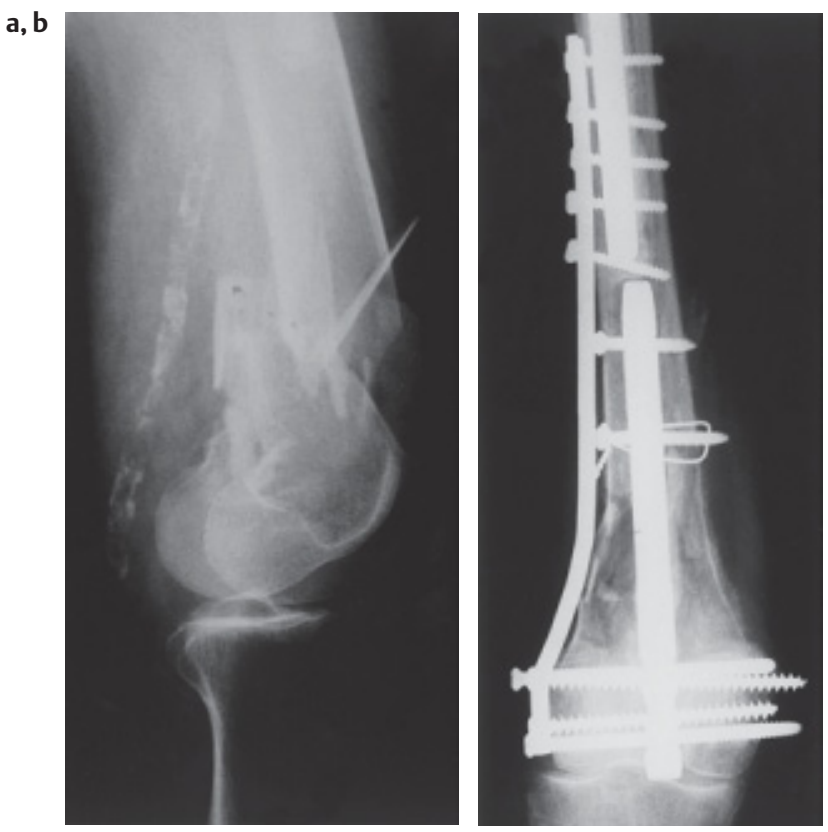

Abb. 5 (a) 81-jährige Patientin nach häuslichem Sturz. (b) Kombination von subkutaner Platte und retrogradem Femurnagel.

durchgeführten Hüfttotalendoprothesen: Seine Empfehlung geht zum Zementieren.

Johansson [13] fand bei 37 Fällen in $40 \%$ zufrieden stellende und $60 \%$ unbefriedigende Ergebnisse.

\section{Eigene Ergebnisse}

Von August 1985 bis Juli 2005 wurden in unserer Klinik 124 Patienten mit einer periprothetischen Fraktur versorgt (Abb.6). Es handelte sich um 31 Männer mit einem Durchschnittsalter von 69,2 Jahren sowie um 93 Frauen mit einem Durchschnittsalter von 77 Jahren. Das Geschlechterverhältnis lag bei $1: 3$ (männlich/weiblich). Bei 60 Patienten fand sich ein stabiles Implantat, in 64 Fällen wurde eine gelockerte Endoprothese diagnostiziert. In 60 Fällen wurde eine extramedulläre Schienung durchgeführt: $48 \times$ mit Platte, $12 \times$ mit dem LISS-System, $40 \times$ mit Spongiosaplastik, $15 \times$ mit Drahtcerclage, in einem Fall mit distal der Prothese gelegenen gekreuzten Kirschner-Drähten. In 6 Fällen erfolgte kombinierte Therapie mit retrogradem Femurmarknagel. In 64 Fällen wurde eine intramedulläre Schienung durchgeführt, $21 \times$ mit Wagner-Schaft, $30 \times$ mit Aesculap-Revisionsschaft mit distaler Verriegelung, $3 \times$ kam eine Krückstockprothese wegen hochgradiger Knochenatrophie im proximalen Schaftbereich zum Einsatz, $1 \times$ eine zementierte Langschaftprothese, $1 \times$ eine zementierte Müller-Gradschaftprothese und $8 \times$ ein retrograder Femurmarknagel (Tab. 2).

Folgendes Behandlungsschema kam zur Anwendung: Bei stabilem Sitz der Prothese wurde eine Plattenosteosynthese, bei gelockerter Prothese eine intramedulläre Schienung mit Revisionsprothese durchgeführt. Probleme ergaben sich hinsichtlich einer vorhandenen Osteopo-

\begin{tabular}{lr}
\hline Tab. 2 Eigene Ergebnisse & \\
\hline $\begin{array}{l}\text { Intramedulläre Schienung }(\mathbf{n = 6 4 )} \\
\text { Wagner-Schaft }\end{array}$ & 21 \\
Revisionsprothese mit distaler & 30 \\
Verriegelung & \\
Krückstockprothese & 3 \\
Retrograder Femurnagel & 8 \\
Zementierter Langschaft & 1 \\
Zementierter Müller-Gradschaft & 1 \\
Extramedulläre Schienung (n=60) & \\
Plattenosteosynthese & 48 \\
LISS-System & 11 \\
Gekreuzte K-Drähte & 1
\end{tabular}




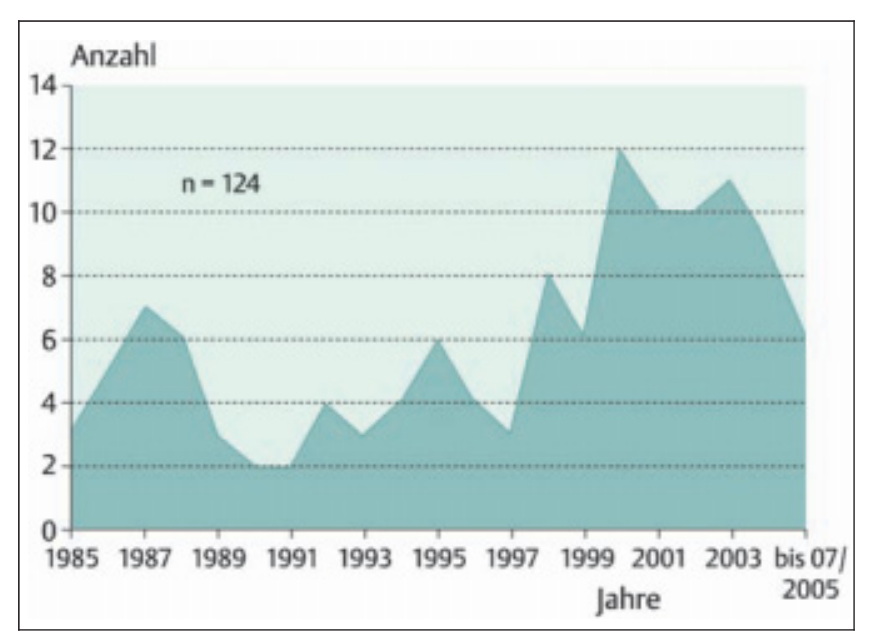

Abb. 6 Anzahl der periprothetischen Frakturen (AKH Celle 1985-2005).
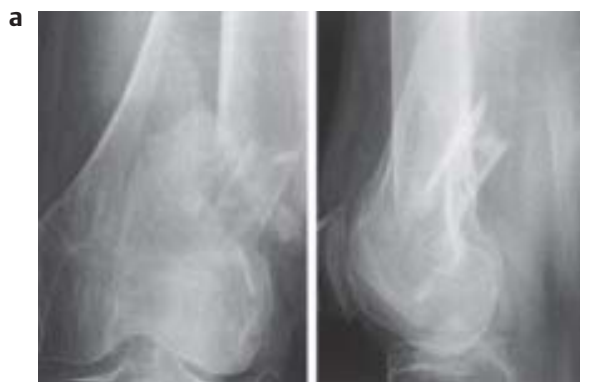

b

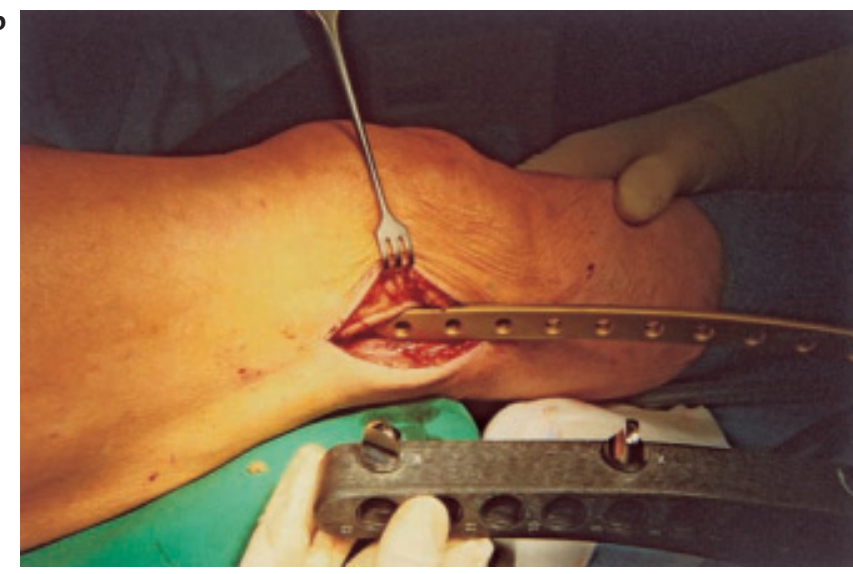

C

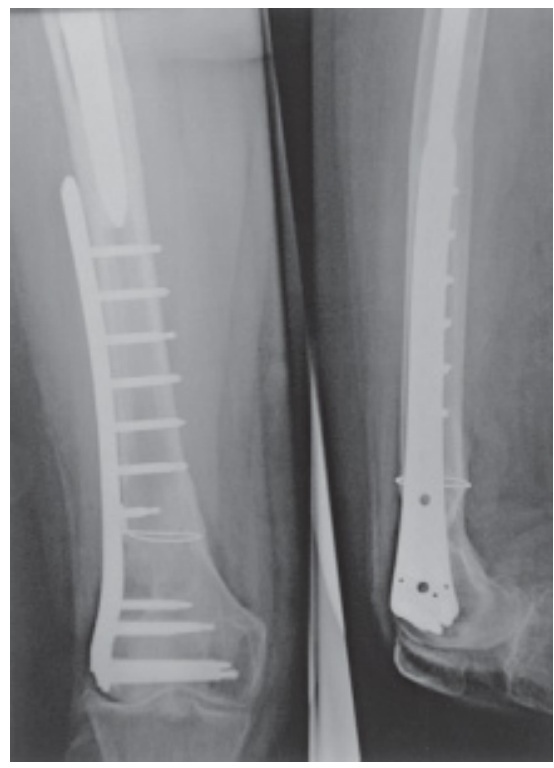

a

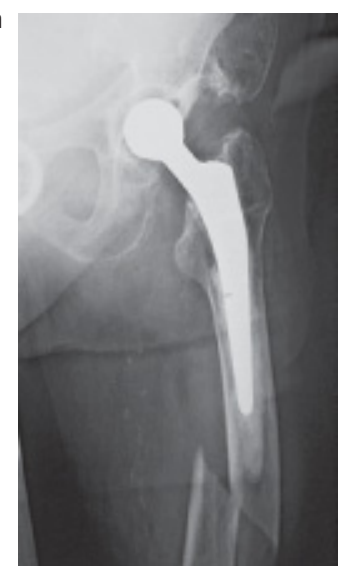

b
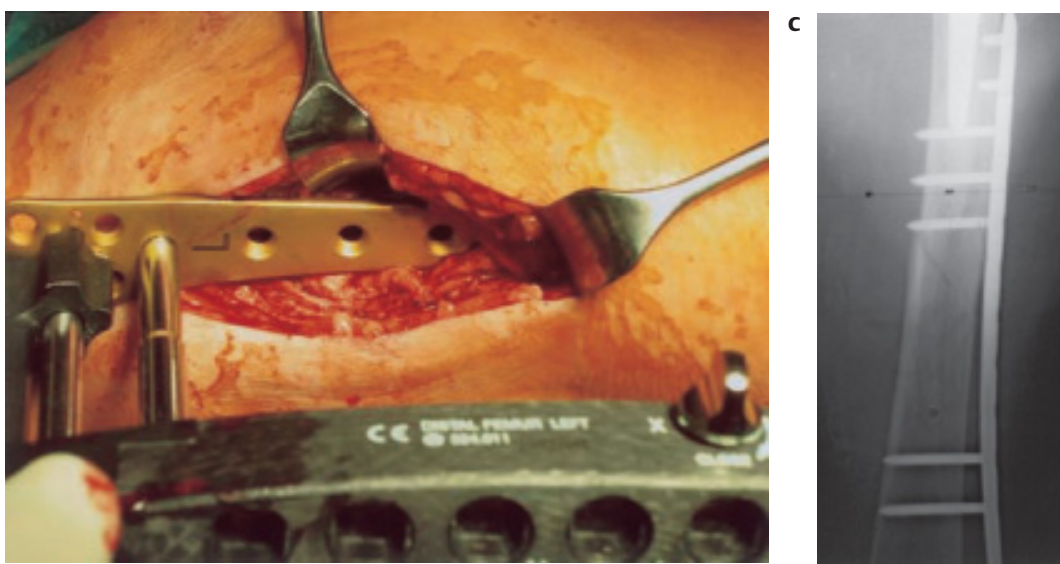

Abb. 8 (a) 82-jährige Patientin, Sturz aus dem Bett, präoperative Röntgenaufnahmen. (b) Anlage der LISS-Platte. (c) Fixation der LISS-Platte in Höhe des Prothesenschaftes mit monokortikalen Schrauben. rose, Compliance, Lebensalter und Begleiterkrankungen der Patienten. Bei der Durchführung einer konventionellen Plattenosteosynthese wurde eine mediale Spongiosaplastik durchgeführt. Beim LISS-System wurde darauf verzichtet. Wichtig sind ausreichend lange Platten mit frakturferner Schraubenapplikation.

An Komplikationen fanden wir 3 Plattenbrüche, die mittels Reosteosynthese und Spongiosaplastik zur Ausheilung gebracht werden konnten. In 2 Fällen wurde ein Endoprothesenwechsel wegen Pseudarthrosen erforderlich. Bei 3 weiteren Patienten kam es zum Einsinken des ger Patient nach Sturz, präoperative Röntgenbilder in 2 Ebenen. (b) Fixation mit eingeschobener LISS-Platte und Cerclage, (c) postoperative Röntgenbilder in 2 Ebenen. 

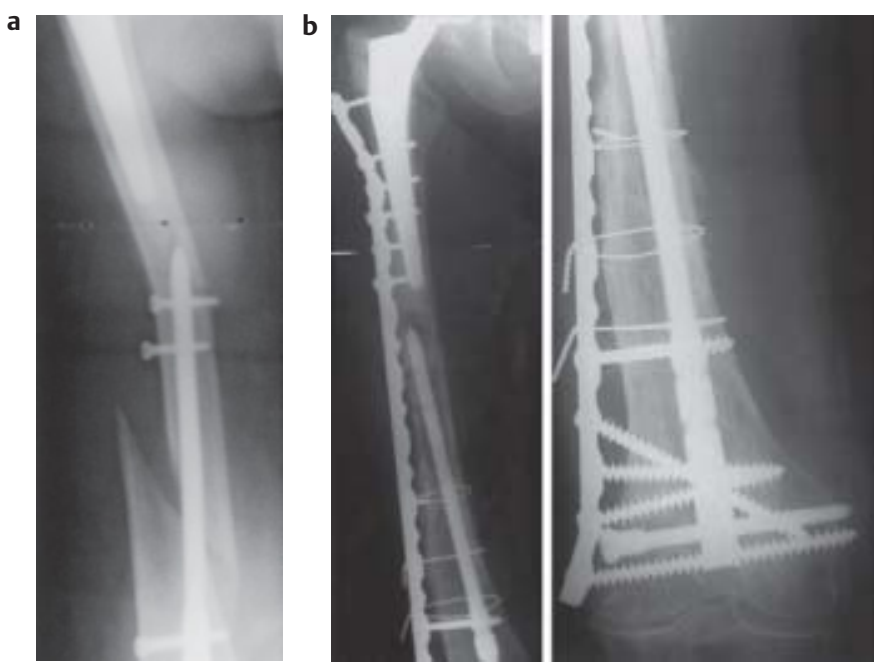

Abb.9 (a) 93-jährige Patientin nach Sturz, präoperative Röntgenaufnahmen. (b) Frakturversorgung mit Platte, Drahtcerclagen und medialer homologer Spongiosaplastik.
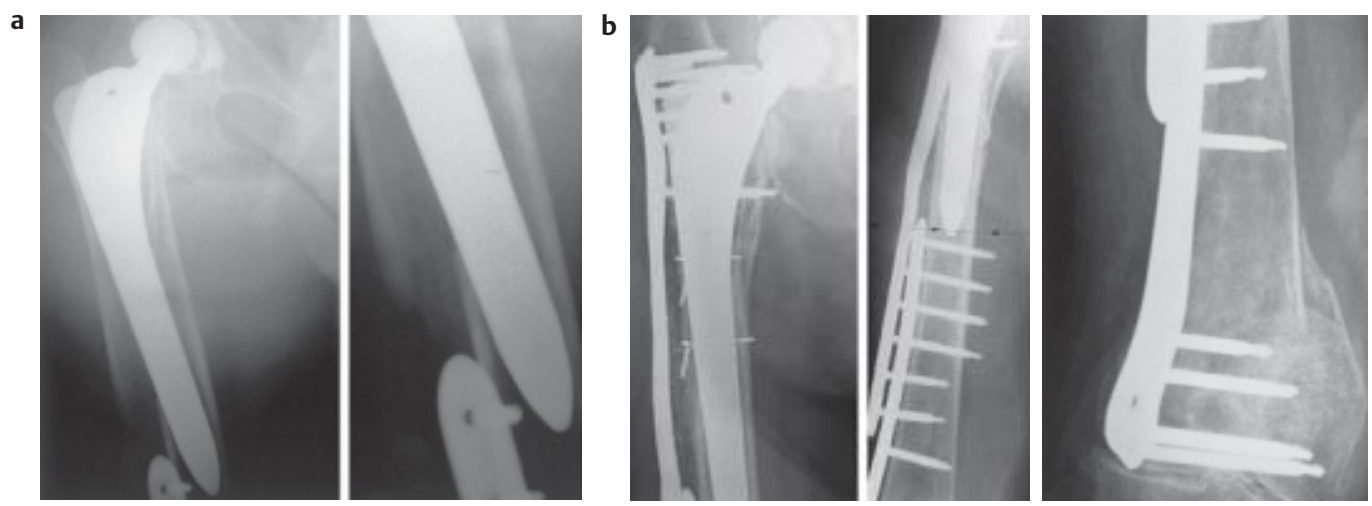

Abb.10 (a) 86-jährige Patientin nach Sturz im Garten. (b) Stabilisation mit umgedrehter LISS-Platte und Cerclagen bei bereits mit LISS versorgter distaler Femurfraktur.
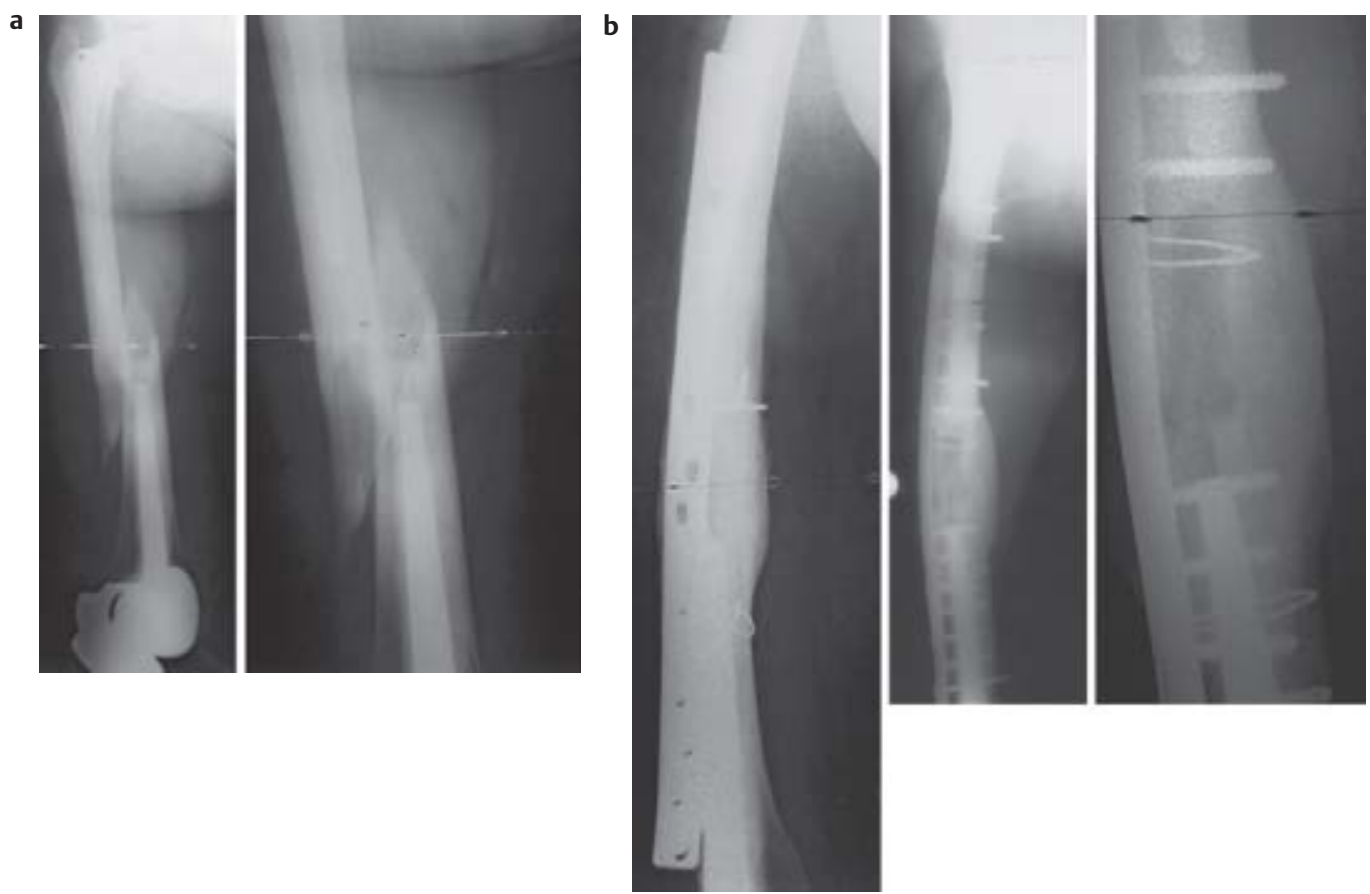

Abb.11 (a) 77-jährige Patientin nach Sturz

Interprothetische Fraktur.

Frakturstabilisation mit Plat-

te und Cerclagen.

Sturz 8 Wochen postoperativ mit Plattenbruch, Reosteo-

synthese und Spongiosaplastik: (b) 1 Jahr danach Frakturheilung. 

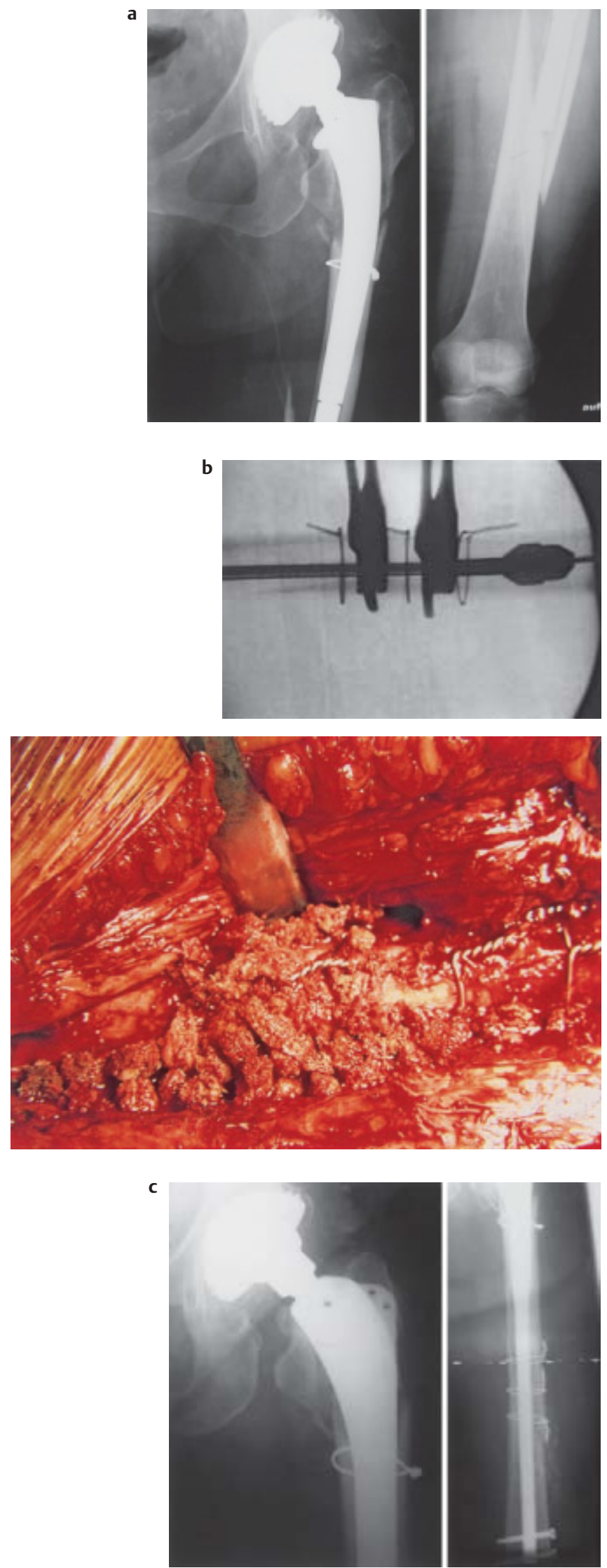

Abb.12 (a) 81-jährige Patientin. Subprothetische Femurfraktur im Anschluss an Schaftwechsel wegen periprothetischer Fraktur, (b) intraoperativ, (c) postoperativ. zementfreien Wagner-Revisionsschaftes, was ohne klinische Relevanz blieb. Bolzenbrüche bei den distal verriegelten Langschaftprothesen konnten wir nicht nachweisen. Bei einer Patientin mit nicht gelockerter Hüftendoprothese und femoral gestielter Knieendoprothese kam es nach Plattenosteosynthese mit Spongiosaplastik zur leichtgradigen varischen Schaftdeformierung ohne Beschwerdesymptomatik.

\section{Präventionsmaßnahmen der periprothetischen Fraktur}

Subtile präoperative Planung, gewebeschonendes Operieren - vor allem bei zementfreier Versorgung - ausreichender operativer Zugang, ggf. intraoperative Zuhilfenahme eines Röntgenbildwandlers bei Revisionseingriffen und anatomischen Besonderheiten, regelmäßige postoperative Nachuntersuchungen der Patienten mit rechtzeitigen Revisionseingriffen - auch bei klinischer Beschwerdefreiheit sowie das Tragen von Hüftprotektoren, vor allem beim älteren Patienten mit ausgeprägter Osteoporose erscheinen empfehlenswert und Erfolg versprechend.

\section{Schlussfolgerung}

Die periprothetische Fraktur wird immer häufiger, sie erfordert das ganze Können des behandelnden Chirurgen. Große Probleme in der chirurgischen Versorgung resultieren durch ständig steigendes Lebensalter der Patienten mit einhergehender Multimorbidität. Die chirurgischen Versorgungsmöglichkeiten sind vielfältig.

Operationszeitpunkt und Auswahl des einzuschlagenden Operationsverfahrens gehören in die Hand eines geübten, erfahrenen Chirurgen bzw. eines Operationsteams.

Voraussetzung ist ein umfangreiches Lager an Implantaten mit abgestimmtem Instrumentarium, Bereitstellung von Fremdblut und Knochen (Knochenbank) sowie die für diese Eingriffe notwendige Infrastruktur. Postoperativ muss eine adäquate krankengymnastische Nachbehandlung gewährleistet sein.

Kleinere Kliniken, die strukturell und personell nicht in der Lage sind, solche zum Teil aufwändigen und schwierigen Operationsverfahren durchzuführen, sollten nach Erstversorgung der Patienten diese zur definitiven Frakturversorgung in entsprechende Zentren verlegen. 


\section{Literatur}

${ }^{1}$ Beals RK, Tower SS. Periprosthetic Fractures of the Femur. Clin Orthop 1996 Jun; 327: 238- 246

2 Berry DJ. Epidemiology: hip and knee. Orthop clin North Am 1999 Apr; 30 (2): $191-207$

${ }^{3}$ Bethea JS. 33d, de Andrade JR, Fleming LL, Lindenbaum SD: Proximal femoral fractures following total hip arthroplasty. Clin Orthop 1982 Oct; 170: 95-106

${ }^{4}$ Bezwada HP, Neubauer P, Baker J, Israelite CL, Johanson NA. Periprosthetic supracondylar femur fractures following total knee arthroplasty. J Arthroplasty 2004 Jun; 19 (4): 453-458

${ }^{5}$ Duncan CP, Masri BA. Fractures of the femur after hip replacement. Instr Course Lect 1995; 44: 293-304

${ }^{6}$ Duwelius PJ, Schmidt AH, Kyle RF, Talbott V, Ellis TJ, Butler JB. A prospective, modernized treatment protocol for periprosthetic femur fractures. Orthop Clin North Am 2004 OctM; 35 (4): $485-492$

${ }^{7}$ Eingartner C, Volkmann R, Putz M, Weller S. Uncemented revision stem for biological osteosynthesis in periprosthetic femoral fractures. Int Orthop 1997; 21 (1): 25-29

${ }^{8}$ Fink B, Fuerst M, Singer J. Periprosthetic fractures of the femur associated with hip arthroplasty. Arch Orthop Trauma Surg 2005 Jul 6

${ }^{9}$ Foster AP,Thompson NW, Wang J, Charlwood AP. Periprosthetic femoral fractures - a comparison between cemented and uncemented hemiarthroplasties. Injury 2005 Mar; 36 (3): 424-429

${ }^{10}$ Gliatis J, Megas P, Panagiotopaulos E, Lambiris E. Midterm results of treatment with a retrograde mail for supracondylar periprosthetic fractures of the femur following total knee artrhoplasty. J Orthop Trauma 2005 Mar; 19 (3): 164-170

${ }^{11}$ Haddad FS, Duncan CP, Berry DJ, Lewallen DG, Gross AE. Periprosthetic femoral fractures around well-fixed implants: use of cortical only allografts with or without a plate. JBJS Am 2002 Jun; 84 (6): 945-950
${ }^{12}$ Hopf C, Hopf T, Rompe JD. Behandlungskonzepte von Oberschenkelfrakturen nach totalem Hüft- oder Kniegelenkersatz: intraoder extramedulläre Stabilisation? Unfallchirurg 1996 Jan; 99 (1): 31 - 37

${ }^{13}$ Johansson JE, McBroom R, Barrington TW, Hunter GA. Fracture of the ipsilateral femur in patients with total hip replacement. JBJS Am 1981 Dec; 63 (9): 1435-1442

${ }^{14}$ Kamineni S, Vindlacheruvu R, Ware HE. Periprosthetic femoral shaft fractures treated with plate and cable fixation. Injury 1999 May; 30 (4): $261-268$

${ }^{15}$ Lowrey CE, Nord KD, Wilson MR, Trick LW. Periprosthetic femur fractures: a complication of rigid cylindrical reaming? Orthop Tran 1991; 15: 342

${ }^{16}$ MacDonald SJ, Paprosky WG, Jablonsky WS, Magnus RG. Periprosthetic femoral fractures treated with a long-stem cementless component. J Arthroplasty $2001 \mathrm{Apr} ; 16$ (3): 379-383

17 Mont MA, Maar DC. Fractures of the ipsilateral femur after hip arthroplasty. A statistical analysis of outcome based on 487 patients. J Arthroplasty 1994 Oct; 9 (5): $511-519$

18 Oestern HJ. 5. Celler AO-Seminar, 12.03.2005 Periprothetische Frakturen

${ }^{19}$ Panasiuk M, Kmieciak M. Treatment of periprosthetic fractures of the distal femur with the LISS system. Chir Narzadow Ruchn Orthop Pol 2004; 69 (6): 369-371

20 Parvizi J, Rapuri VR, Purtill JJ, Shayrkey PF, Rothman RH, Hozack WJ. Treatment protocol for proximal femoral periprosthetic fractures. JBJ Am 2004 Mar; 86 A: 8-16

${ }^{21}$ Schwartz JT jr, Mayer JG, Engh CA. Femoral fracture during non-cemented total hip arthroplasty. JBJS 1989; 71: 1135-1142

22 Scott RD, Turner RH, Leitzes SM, Aufranc OF Femoral fractures in conjunction with total hip replacement. JBJS Am 1975 Jun; 57 (4): 494-501

${ }^{23}$ Siegmeth A, Menth-Chiari WA, Wozasek GE, Vecsei V. Femur fractures in patients with hip arthroplasty: indication for revision arthroplasty. J South Orthop Assoc 1998; (4): $251-258$
${ }^{24}$ Spitaler R, Reichetseder J, Rappold G, Leixnering $M$, Hertz H. Periprothetische Femurfraktur bei ipsilateraler Hüft- bzw. Knieendoprothese: Das Akutversorgungskonzept - ein operativer Algorithmus. Akt Traumatol 2003; 33: 272 - 280

${ }^{25}$ Tsiridis E, Narvani AA, Haddad FS, Timperley JA, Gie GA. Impaction femoral allografting and cemented revision for periprosthetic femoral fractures. JBJS $\mathrm{Br} 2004$ Nov; 86 (8): $1124-1132$

${ }^{26}$ Wagner M. Die Markangelung der periprothetischen Femurfraktur mit der Femur-Revisionsprothese. Akt Traumatologie 2003; 33: $281-283$

${ }^{27}$ Wahl B, Grasshoff H, Meinecke I, Neumann HW. Ergebnisse der operativen Therapie periprothetischer Femurschaftfrakturen bei Hüfttotalendoprothesen. Zentralbl Chir 2001 Jan; 126 (1): $50-54$

${ }^{28}$ van der Wal BC, Vischjager M, Grimm B, Heyligers IC, Tonio AJ. Periprosthetic fractures around cementless hydroxyapatitecoated femoral stems. Int Orthop 2005 Aug; 29 (4): 235-240

${ }^{29}$ Wilson D, Frei H, Masri BA, Oxland TR, Duncan CP. A biomechanical study comparing corrical onlay allograft struts and plates in the treatment of periprosthetic femoral fractures. Clin Biomech (Bristol, Avon) 2005 Jan; 20 (1): $70-76$

\section{Dr. med. Herbert Hessler Oberarzt}

Prof. Dr. med. Hans-Jörg Oestern Chefarzt

Klinik für Unfall- und Wiederherstellungschirurgie Allgemeines Krankenhaus

Siemensplatz 4

D-29223 Celle 